Abstract

\title{
Accurate 3D Face and Body Scanning Using an Irritation-Free Pattern Projection System ${ }^{\dagger}$
}

\author{
Christian Bräuer-Burchardt 1,*, Anika Brahm ${ }^{1}$, Stefan Heist ${ }^{1}$, Patrick Dietrich ${ }^{1}$, Peter Kühmstedt ${ }^{1}$ \\ and Gunther Notni 1,2 \\ 1 Fraunhofer Institute for Applied Optics and Precision Engineering, Albert-Einstein-Str. 7, D-07745 Jena, \\ Germany; anika.brahm@iof.fraunhofer.de (A.B.); stefan.heist@iof.fraunhofer.de (S.H.); \\ patrick.dietrich@iof.fraunhofer.de (P.D.); peter.kuehmstedt@iof.fraunhofer.de (P.K.); \\ gunther.notni@iof.fraunhofer.de (G.N.) \\ 2 Department of Mechanical Engineering, Technical University Ilmenau, Ehrenbergstraße 29, \\ D-98693 Ilmenau, Germany \\ * Correspondence: christian.braeuer-burchardt@iof.fraunhofer.de \\ + Presented at the 5th International Symposium on Sensor Science (I3S 2017), Barcelona, Spain, \\ 27-29 September 2017.
}

Published: 11 December 2017

Three-dimensional scanning of human bodies or body parts is gaining increasing importance in applications where moving people need to be captured. This could be, for example, people tracking, lip reading, or gesture detection.

The advantages of optical 3D sensing in these fields are the contactless measurement and the high potential concerning speed and measurement accuracy. However, classical illumination by structured light may disturb personal activities. In this paper, a new $3 \mathrm{D}$ scanning system is introduced which is absolutely irritation-free based on the structured light illumination by aperiodic sinusoidal fringe patterns in the near infrared range. Hence, it is particularly suitable for continuing human face scanning. The illumination system is based on the GOBO principle.

Applications interpreting human facial expressions or gestures often require a very short latency time of the data capture because of the necessary subsequent processing of the 3D point clouds. The presented 3D scanner setup achieves a latency time of approximately $100 \mathrm{~ms}$ until the $3 \mathrm{D}$ data of a $4 \mathrm{MPx}$ scan is available.

Examples of the acquisition of sequences of face and body scans are given.

The measurement accuracy was evaluated by experiments and will be presented and discussed. An outlook of future work is given.

Conflicts of Interest: The authors declare no conflict of interest.

(C) 2017 by the authors. Licensee MDPI, Basel, Switzerland. This article is an open access article distributed under the terms and conditions of the Creative Commons Attribution (CC BY) license (http://creativecommons.org/licenses/by/4.0/). 